\title{
Robust Modeling of Geodetic Altitude from Barometric Altimeter and Weather Data
}

\author{
Maximilian Simonetti and Omar García Crespillo \\ Institute of Communications and Navigation, German Aerospace Center (DLR)
}

\section{BIOGRAPHY}

Maximilian Simonetti received in 2021 his Master's in Aerospace Engineering the Technical University of Munich. Since the same year he is a scientific employee of the Institute for Communications and Navigation of the German Aerospace Center (DLR), where he researches about the integrity of multi-sensor navigation architectures.

Omar García Crespillo is the leader of the Integrated Navigation Systems Integrity group at the Navigation department of the German Aerospace Center (DLR), where he works since 2013. He holds a M.Sc. in Telecommunication Engineering from the University of Malaga in Spain. His current field of research includes multi-sensor fusion algorithms, GNSS, inertial sensors and integrity monitoring for safe ground and air transportation systems.

\begin{abstract}
Vertical navigation is crucial for safe aircraft separation, which have been traditionally based on flight altitude levels. This altitude information is normally called standard pressure altitude since it is computed from airborne pressure measurements performed by barometers and is referenced to the International Standard Atmosphere (ISA) Mean Sea Level (MSL) isobar surface.

On the other hand, robust geodetic altitude navigation is fundamental for airport nearness operations with tighter requirements and plays a key role for new applications like Urban Air Mobility (UAM). However, the deviations of standard pressure altitude from true geodetic altitude can reach up to several hundreds of meters and therefore its application is limited to relative vertical navigation. The barometric measurements can nevertheless still be used to determine geodetic altitude if additional weather information like pressure and temperature is available and some transformations are applied.

This paper first presents a methodology to compute geodetic altitude from a corrected pressure altitude obtained with airborne pressure measurements and external weather data. Flight data is used to assess the achievable geodetic altitude accuracy from 20 flight hours with the German Aerospace Center (DLR) Dassault Falcon 20-ES aircraft. Second, one linear model is derived to mitigate residual errors that dependent on flight dynamics. Finally and based on the available flight data, first robust stochastic error models are proposed to support the adoption of barometric pressure measurements for safe geodetic altitude navigation. In particular, two models are derived. The first one provides a Gaussian overbound for the computed geodetic altitude so that it can be used either directly as altitude information or in combination with other sensors in snapshot (e.g., least-squares) estimators. The second is a dynamic model that bounds the power spectral density of the error with a first-order Gauss Markov process. In this way this model can be easily incorporated in a sequential estimator, like a Kalman filter, and in combination with other sensors.
\end{abstract}

\section{INTRODUCTION}

Vertical navigation is essential for safe Air Traffic Management (ATM). In particular, vertical separation between aircraft above transition altitudes is ensured by the definition of flight levels, which are based on standard pressure altitude [1]. Below transition altitudes, it is necessary to have the relative altitude with respect to the ground, this is achieved by adjusting the pressure altitude to sea level (also called QNH) by using airport reference weather information [2]. Both standard pressure and QNH altitude deviate from geometric altitude above Mean Sea Level (MSL). Vertical navigation based on geodetic altitude, instead, is crucial for new aircraft operations like precision approaches and automatic landing [3], which are foreseen to be primary based on Global Navigation Satellite Systems (GNSS). In the case of GNSS service disruption, Alternative Positioning Navigation and Timing (APNT) services based on ground radionavigation signals like DME [4], LDACS [5] and possibly other sensors and systems [6.7], can provide horizontal navigation. However, it has been shown that for APNT algorithms to converge, accurate geodetic altitude information is fundamental [8, 9]. Furthermore, new Urban Air Mobility (UAM) applications, which are expected to operate closer to the ground, will need safe geodetic altitude information for navigation, guidance, collision avoidance and unmanned traffic management (UTM) [10].

Navigation algorithms based on INS integration with GNSS are able to provide geodetic altitude information. However, the 


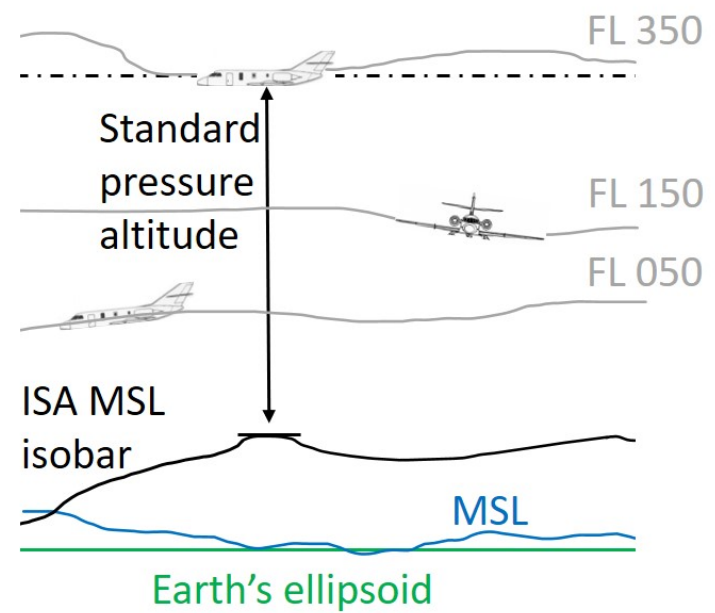

Figure 1: FLight levels, standard pressure altitude and various altitude references

inherent instability of the INS vertical channel [11] and the poorer performances of GNSS in vertical domain than in the horizontal one [12], along with the numerous threats to GNSS integrity, reduce the reliability of their provided geodetic altitude information in some situations. Depending on the application, barometers can be considered as an additional sensor for geodetic altitude navigation. The use of barometric altimeters for this purpose requires rigorous conversions to geodetic altitude since both in the case of standard and corrected pressure altitude, remarkable differences between the values of pressure altitude and the ones of true geodetic altitude are to be expected. This is due to the fact that these two altitudes differ both in regard to their references and their scales. Furthermore, the atmosphere does not exactly behave as in standard models.

Previous work related to the adaptation of pressure measurements to geodetic altitude considers an adjustment of the barometric pressures during a whole flight so that the corrected pressure altitude equals the geodetic altitude at take-off [13]. The authors in [13] additionally estimate various error model parameters with a least-square algorithm to correct pressure altitude with respect to GPS altitude. The RMS of the residual errors is however still in the order of hundreds of meters. The authors in [14] consider the addition of geoid undulations to pressure altitude before incorporating it both into a Kalman filter (KF) and a Weighted-Least-Square estimator together with GPS altitude. In [15], pressure altitude is merged with GPS without any consideration of bias or scale factors. In [11] the author describes an integration scheme of pressure and geodetic altitude based on the estimation of a bias and a scale factor term. However, the methodology and the specific values that are used to model the barometric measurement errors are not mentioned in any of the previous work. In [16], the authors provide a model for barometric altimeters where pressure altitude is corrected with weather data provided by ground stations. The authors consider geoid undulations but not the difference in scale to finally provide altitude information. The altitude information is then merged with GPS altitude augmented by WAAS (Wide Area Augmentation System). Time-correlated error sources are therefore not covered by this model. Furthermore, the analysis of [16] are based on meteorological observation data and not on measurements collected during flights. In [17], the authors provide vertical position error bounding for UAVs (Unmanned Aerial Vehicles) using corrected pressure altitude. This error bound is then used within a WLS (weighted least square) RAIM for the integration of baroaltimeter with GPS. A complete conversion of the corrected pressure altitude to geodetic altitude is missing in this work too. Besides, by deriving the overbounding model the difference between the geopotential and the geometric scales was not considered, probably because of the considerably low altitude above MSL. Other approach to obtain geometric altitude above MSL is based on the use of pressure and temperature measurements within an iterative algorithm, known as Blanchard algorithm [18, 19]. The resulting altitude however presents an error that increases over time.

This paper aims at analyzing the benefit of using external weather data on the computation of pressure altitude. After proper translation of scale and reference, geodetic altitudes can be obtained with few meters of error based on the analysis of several hours of flight data with the German Aerospace Center (DLR) Dassault Falcon aircraft. Apart from possible inaccuracies of weather data, it is found that the flight dynamics have an impact in the static ports of the barometer sensor. A linear model is then propose to mitigate these type of errors based on the aircraft attitude. The final residual errors in altitude can be stochastically modelled to support the robust integration of this altitude with other sensors or systems. In particular, a robust error model is derived for snapshot algorithms based on paired overbounding. Then, a dynamic error model is obtained by analysing the power spectral density (PSD) of the residual error over the expected stationary phases. This model can be incorporated in Kalman filtering for the integration with additional systems, like INS/GNSS-based navigation systems [2]. 
This paper is organized as follows. Section $\Pi$ explains the different types of altitudes that are necessary for the pressure to geometric altitude transformation. Section IIII reviews how pressure altitude is traditionally computed based on pressure measurements, with the particular case of standard pressure altitude. In Section [IV] the methodology to obtain geodetic altitude from pressure measurements and weather data is explained. Then, Section V $\mathrm{p}$ provides with the experimental flight information and the geodetic altitude results. This is followed by the methodology and derivation of robust snapshot and dynamic error models in Section VI Finally, Section VII closes the paper with the importance of this work for safe navigation systems.

\section{ALTITUDE DEFINITIONS}

An altitude is a vertical distance between an object or vehicle to a certain reference surface. The increment of the altitude with respect to distance is given by a certain scale. Figure 2 shows different altitude definitions according to some possible reference surfaces and altitude scales.

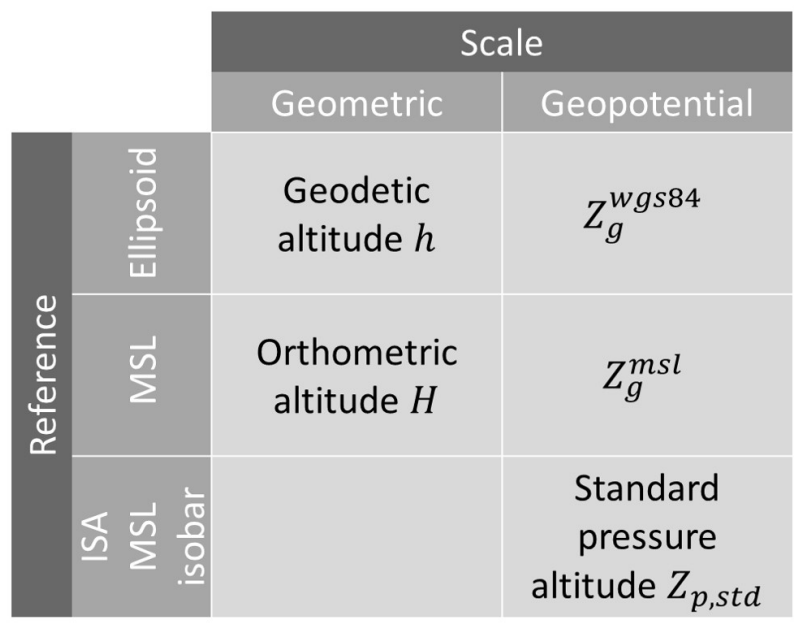

Figure 2: Different altitude definitions

Geodetic altitude $h$ is the distance from a given point to an Earth's reference ellipsoid along the normal line to this surface. In this work, we consider the WGS-84 ellipsoid as the reference for the target geodetic altitude since it is nowadays the most widely used for navigation purposes.

Another possible reference surface is the geoid, which is a model of the Earth's surface defined as the equipotential surface of the Earth's gravity field which best fits, in a least squares sense, global mean sea level (MSL) [20]. This is the level of the oceans averaged over the tide cycle. Since water tends to maintain a constant potential energy, it is expected that the geoid coincides with the MSL with around 1 meter difference worldwide [20]. The distance from a point to the geoid surface along the normal to this surface is typically called orthometric altitude or altitude above mean sea level, herein denoted by $H$. This is related to the geodetic altitude through

$$
H \approx h-N(L, \lambda) .
$$

The geoid undulation $N$ is the distance of the geoid referenced to the ellipsoidal surface for a given latitude and longitude. The geoid undulation is positive where the geoid surface is outside the ellipsoid and ranges worldwide between approximately -105 and 85 meters, as shown in Figure 3b Geodetic and orthometric altitudes are both geometric altitudes, because of their geometric scale. On the other hand, by using a geopotential scale an altitude with respect to the MSL surface can be defined as [21]:

$$
Z_{g}^{m s l}(L, \lambda, H)=\frac{1}{g_{0}} \int_{0}^{H} g(L, \lambda, z) d z
$$

where $g_{0}$ is the average MSL acceleration due to gravity. Similarly, a geopotential altitude with respect to a reference ellipsoid can be also defined from a geodetic altitude as [21]:

$$
Z_{g}^{w g s 84}(L, h)=\frac{1}{g_{0}} \int_{0}^{h} \gamma_{h}(L, z) d z
$$




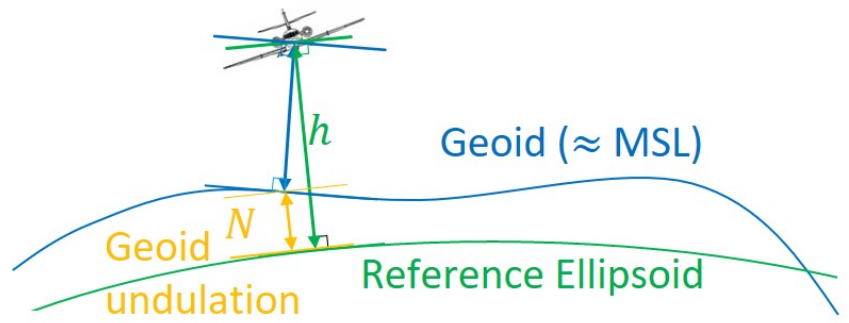

(a) Relationship between geodetic and orthometric altitudes

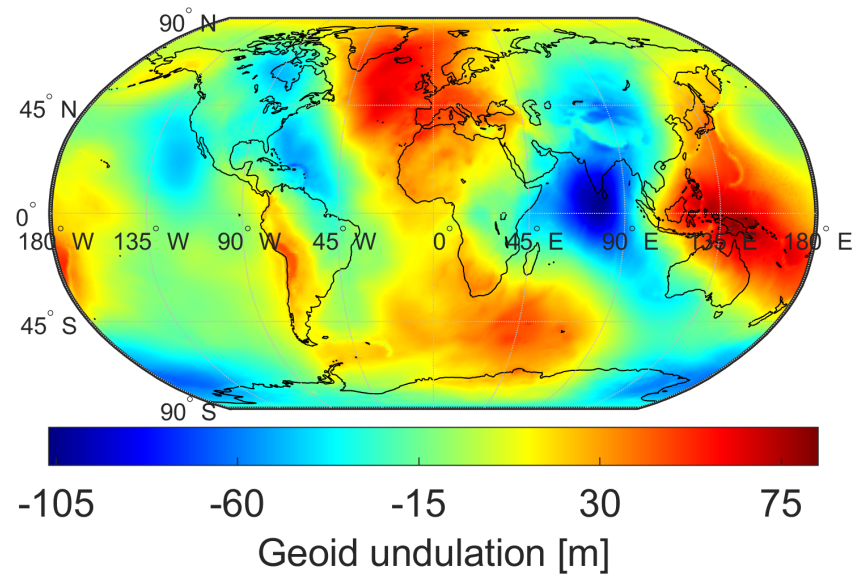

(b) Geoid undulations obtained from the EGM96 Earth Geopotential Model

Figure 3: Geodetic and orthometric altitudes and the Geoid

where $\gamma_{h}$ denotes the gravity at a given geodetic altitude. A close form solution of Equation $(3)$ is provided in [11]:

$$
Z_{g}^{w g s 84}(L, h)=\frac{\gamma(L)}{g_{0}} h\left[1-\frac{h}{R_{e}}\left(1+f+m-2 f \sin ^{2} L\right)+\frac{h^{2}}{R_{e}^{2}}\right] .
$$

The gravity at the ellipsoidal surface $\gamma(L)$ according to the Somigliana model is [21]:

$$
\gamma(L)=\frac{R_{e} \gamma_{e} \cos ^{2} L+R_{p} \gamma_{p} \sin ^{2} L}{\sqrt{R_{e}^{2} \cos ^{2} L+R_{p}^{2} \sin ^{2} L}},
$$

where $R_{\mathrm{e}}$ and $R_{\mathrm{p}}$ denote the WGS84 equatorial and polar radii, respectively, while $\gamma_{\mathrm{e}}=9.7803253359 \mathrm{~ms}^{-2}$ and $\gamma_{\mathrm{p}}=$ $9.8321849378 \mathrm{~ms}^{-2}$ are the equatorial and polar gravity accelerations at the ellipsoidal surface, respectively. Further terms in Equation (4) are defined as [21]:

$$
f=\frac{R_{e}-R_{p}}{R_{e}} \text { and } m=\Omega^{2} \frac{R_{e}^{2} R_{p}}{G M},
$$

where $\Omega$ and $G M$ are the Earth's angular velocity and gravitational constant, respectively.

Figure 4 shows the difference between geodetic altitude $h$ and $Z_{\mathrm{g}}^{\mathrm{wgs} 84}$ as a function of latitude and geodetic altitude. This figure expresses the difference between the geometric and the geopotential scales since both altitudes are referenced to the WGS84 surface. Geopotential and ellipsoidal altitudes $Z_{\mathrm{g}}^{\mathrm{wgs} 84}$ can be converted to geopotential altitudes with respect to MSL $Z_{\mathrm{g}}^{\mathrm{msl}}$ with the following relation [21]:

$$
Z_{g}^{m s l}(L, \lambda, H)=Z_{g}^{w g s 84}(L, h)-Z_{g}^{w g s 84}(L, N(L, \lambda)),
$$

where $Z_{g}^{w g s 84}(L, N(L, \lambda))$ is the geopotential altitude of the geoid above the ellipsoid. This expression is important when using weather data, since altitude information provided by weather services is normally given in geopotential scale above MSL. 


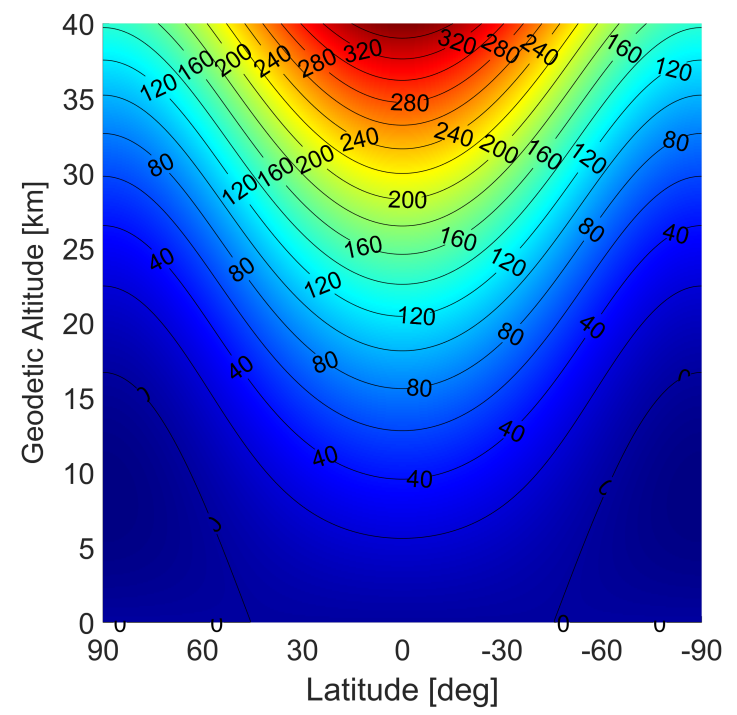

Figure 4: Difference between geometric and geopotential altitudes above WGS84 (reproduced with the same style adopted in [20])

\section{STANDARD PRESSURE ALTITUDE}

Barometric or pressure altitude can be obtained from measurements from a barometric sensor. Considering the atmosphere as a perfect gas and under air static equilibrium assumption, the relation between pressure and pressure altitude can be expressed by the following differential equation [22]:

$$
d Z_{g}^{m s l} g_{0}=\frac{-R_{d r y} T}{p} d p
$$

where $d p$ is the vertical change of static air pressure, $\rho$ is the air density, $d H$ is the change in orthometric altitude and $R_{\mathrm{dry}}$ denotes the dry air's gas constant, whose value is reported in Table 1. The ISA model is a static atmospheric model of how temperature and pressure varies over altitude. By using the static ISA model constants, the differential Equation (8) can be solved analytically. The evolution of temperature with respect to geopotential altitudes ranging between 0 and $20 \mathrm{~km}$ can be described as [23]:

$$
\begin{array}{ll}
T_{I S A}=T_{0}-\alpha Z_{g}^{m s l}, & \text { for } 0<Z_{g}^{m s l} \leq 11 \mathrm{~km}, \\
T_{I S A}=T_{1}, & \text { for } 11<Z_{g}^{m s l} \leq 20 \mathrm{~km} .
\end{array}
$$

The temperature lapse rate $\alpha$, and $T_{1}$ are given in Table 1 . Using Equation (9) in Equation (8) leads to:

$$
\begin{array}{ll}
d Z_{g}^{m s l} g_{0}=\frac{-R_{d r y}\left(T_{0}-\alpha Z_{g}^{m s l}\right)}{p} d p, & \text { for } 0<Z_{g}^{m s l} \leq 11 \mathrm{~km}, \\
d Z_{g}^{m s l} g_{0}=\frac{-R_{d r y}\left(T_{1}\right)}{p} d p, & \text { for } 11<Z_{g}^{m s l} \leq 20 \mathrm{~km} .
\end{array}
$$

Table 1: International Standard Atmosphere (ISA) model constants

\begin{tabular}{|c||c|}
\hline ISA Constants & Values \\
\hline$p_{0}$ & $101.325 \times 10^{3} \mathrm{~Pa}$ \\
\hline$T_{0}$ & $288.15 \mathrm{~K}$ \\
\hline$T_{1}$ & $216.65 \mathrm{~K}$ \\
\hline$\alpha$ & $6.5 \mathrm{~K} \mathrm{~km}^{-1}$ \\
\hline$R_{\text {dry }}$ & $287.05287 \mathrm{~J} \mathrm{~kg}^{-1} \mathrm{~K}^{-1}$ \\
\hline
\end{tabular}


Integrating Equation (10) along $Z_{\mathrm{g}}^{\mathrm{msl}}$ from a certain reference geopotential altitude above MSL $Z_{\mathrm{g}, \mathrm{ref}}^{\mathrm{ml}}$ results in:

$$
\begin{array}{ll}
Z_{g}^{m s l}=\frac{T_{r e f}}{\alpha}\left[1-\left(\frac{p}{p_{r e f}}\right)^{\frac{k R_{d r y}}{g_{0}}}\right]+Z_{g, r e f}^{m s l}, & \text { for } 0<Z_{g}^{m s l} \leq 11 \mathrm{~km}, \\
Z_{g}^{m s l}=11 \mathrm{~km}-\frac{T_{11 k m} R_{d r y}}{g_{0}} \ln \left(\frac{p}{p_{11 k m}}\right), & \text { for } 11<Z_{g}^{m s l} \leq 20 \mathrm{~km} .
\end{array}
$$

In Equation (11), $T_{r e f}$ and $p_{\text {ref }}$ denote the temperature and pressure at $Z_{\mathrm{g}, \text { ref }}^{\mathrm{msl}}$, respectively, while $T_{11 \mathrm{~km}}$ and $p_{11 \mathrm{~km}}$ are the temperature and pressure at $Z_{\mathrm{g}}^{\mathrm{msl}}=11 \mathrm{~km}$.

The standard pressure altitude used in aviation is obtained from pressure measurements $p$ with Equation (11) by using as the reference isobar surface the International Standard Atmosphere (ISA) MSL [2]. This consists of setting $Z_{\mathrm{g}, \mathrm{ref}}^{\mathrm{wg}}=0, T_{r e f}=T_{0}$ and $p_{\text {ref }}=p_{0}$. These values of temperature and pressure are reported in Table 1 . In this case, Equation (11) reduces to:

$$
Z_{p, s t d}=\frac{T_{0}}{\alpha}\left[1-\left(\frac{p}{p_{0}}\right)^{\frac{k R_{d r y}}{g_{0}}}\right] .
$$

Standard pressure altitude is therefore an estimate of the altitude above the $p_{0}$ isobar surface. The true pressure and temperature at MSL deviates from the ISA model, and therefore the standard pressure altitude will deviate in proportion from the true geopotential altitude above MSL. Morevoer, pressure altitude could still differ remarkably from the true geopotential altitude above MSL even if the actual MSL values for temperature and pressure are known. This is due to the ISA model approximation of the true atmosphere behavior used for temperature.

\section{GEODETIC ALTITUDE FROM PRESSURE MEASUREMENTS AND WEATHER DATA}

Conversion from the different altitude definitions shown in Figure 2 is possible by applying transformations on the reference surface and altitude scales and if more precise knowledge about pressure and temperature in the atmosphere is available. The next sections show in two steps, how a conversion from standard pressure altitude to geometric altitude is possible by using external weather data.

\section{Weather-corrected Pressure Altitude}

We define weather-corrected pressure altitude $Z_{\mathrm{p}, \mathrm{w}}$ as the aircraft's geopotential pressure altitude above MSL obtained with weather data. It is obtained from Equation (11) when the actual pressure and temperature at the aircraft's horizontal and geopotential altitude location above MSL are used. In the case of an aircraft flying below $11 \mathrm{~km}$ of geopotential altitude above MSL, the second of the two expressions in Equation (11) is used and the actual pressure and temperature values at $11 \mathrm{~km}$ are used.

Estimates of the actual values of weather conditions may be obtained from numerical weather predictions (NWP) services for real-time navigation or from climate reanalyses for offline applications. In this work, we consider the use of climate reanalysis data for the computation of weather-corrected pressure altitude using data from the European Union's Copernicus programme. It can be publicly extracted from the European Centre for Medium Range Weather Forecasts (ECMWF) ERA5 climate reanalysis on pressure levels [24]. This provides data for atmospheric quantities on 37 pressure (i.e. isobar) levels from 1000 to $1 \mathrm{hPa}$. Data is given at each UTC full hour on a global regular 0.25 degrees resolution latitude-longitude grid. The weather dataset can be visualized as a four-dimensional (4D) grid of pressure level, latitude, longitude and time, as shown in Figure 5. For each of the points of this $4 \mathrm{D}$ grid, among the various quantities, the temperature and the geopotential altitude above MSL are reported. 


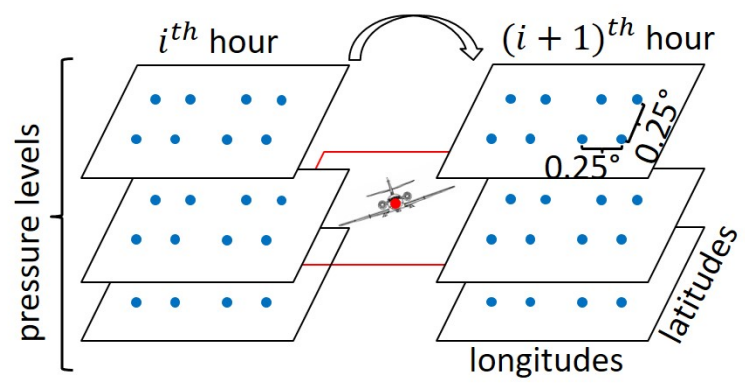

Figure 5: ERA5 weather data structure scheme and the aircraft's position at a certain location and time

Multidimensional interpolation of the ERA5 data is necessary to obtain the temperature and pressure at the aircraft's location. In particular, linear interpolation was applied both in the time domain and in the horizontal plane. For the vertical channel, linear interpolation was performed for temperature, while logarithmic interpolation was applied with respect to pressure [22].

\section{Conversion from Weather-corrected Pressure Altitude to Geodetic Altitude}

Weather-corrected pressure altitude is an estimate of the true geopotential altitude above MSL. Figure 2 shows that if this is known, then the corresponding geodetic altitude may be obtained by applying a two-steps conversion. First, the reference needs to be shifted from the MSL to the reference ellipsoid and secondly the scale has to be converted from the geopotential to the geometric one. In order to shift the reference from MSL to the WGS84 ellipsoid, we can solve for $Z_{g}^{w g s 84}$ in Equation (7) and substitute $Z_{g}^{m s l}$ with the weather corrected pressure altitude $Z_{p, w}$. This yields the estimate $\hat{Z}_{g}^{\text {wgs } 84}$ of the true geopotential altitude above the ellipsoid. Then, Equation (4) can be used to convert from the geopotential scale to the corresponding geodetic altitude. Equation (4) can be solved for example numerically by using close initial approximate altitude (e.g., previous altitude or standard pressure altitude):

$$
\hat{h}=\underset{h}{\arg \min }\left(\left|\hat{Z}_{g}^{w g s 84}-Z_{g}^{w g s 84}(h)\right|\right)
$$

where $\hat{Z}_{g}^{w g s 84}$ is the precomputed weather corrected altitude from pressure measurements and $Z_{g}^{\text {wgs } 84}(h)$ is Equation (4).

\section{RESULTS OF GEODETIC ALTITUDE COMPUTATION FROM PRESSURE MEASUREMENTS}

\section{Experimental Flight Data}

This work is based on the analysis of pressure altitude measurements over nine different flights with DLR's Dassault Falcon 20-E5 aircraft (Figure 6) between the $9^{\text {th }}$ and the $13^{\text {th }}$ of July 2018. The geodetic altitude profiles of these flights are shown in Figure 7 and the horizontal trajectories for each of the flights are shown in Figure 8.

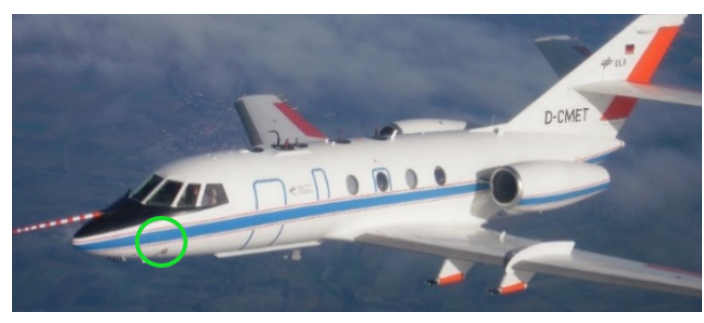

(a) DLR's Dassault Falcon 20-E5

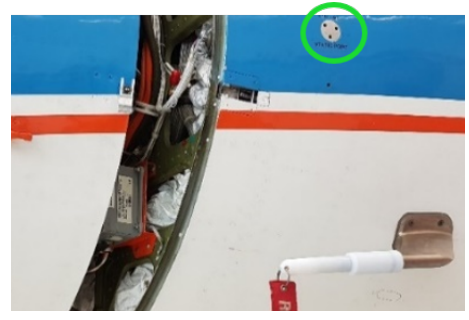

(b) Left static pressure port and Pitot tube

Figure 6: DLR's Dassault Falcon 20-E5's static pressure ports (circled in green) 


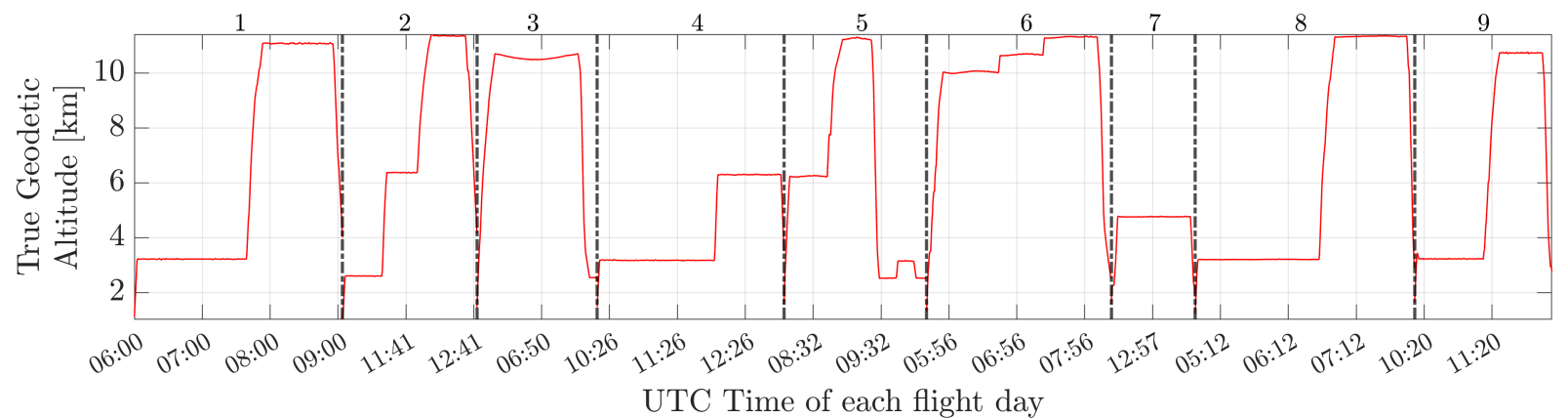

Figure 7: True geodetic altitude profiles of the nine investigated flights

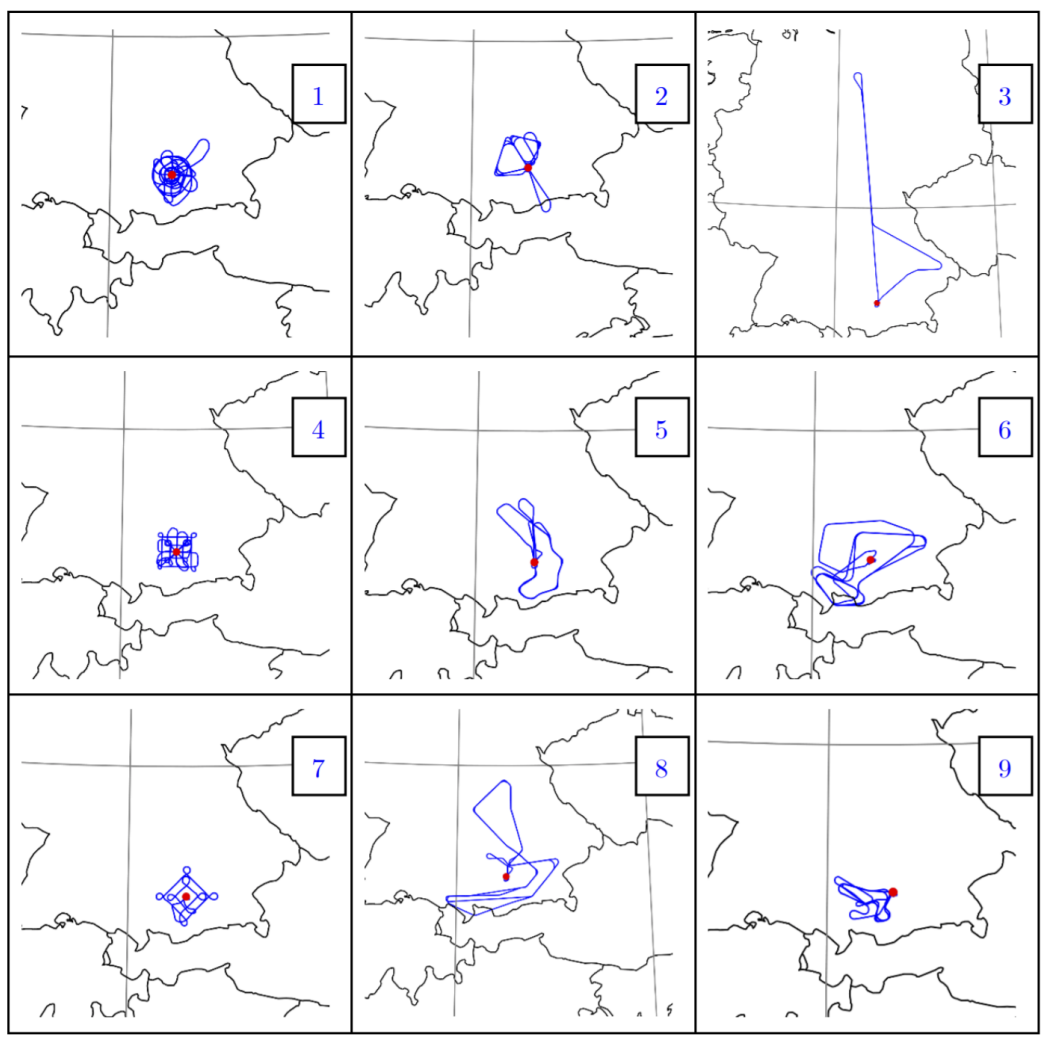

Figure 8: Test flight trajectories projected on the Earth's surface (blue) and Oberpfaffenhofen airport (red)

In particular, a total of approximately 20 hours of pressure altitude measurements at $10 \mathrm{~Hz}$ were recorded. GNSS data was recorded in parallel and it is used after precise post-processing as ground truth for the analysis of the results.

\section{Geodetic Altitude Computation Accuracy}

Figure 9 depicts in black the deviations from the true geodetic altitude of the standard pressure altitude. The error of the computed geodetic altitude is also shown in the same figure in blue. The computed geodetic altitude approximates with a much higher accuracy the true geodetic altitude than the standard pressure altitude. A comparison of Figure 9 with Figure 7 shows a certain level of modulation between the standard pressure's deviations with the true geodetic altitude profiles. In particular, it can be noticed that these deviations generally increase with increasing flight altitude. Nevertheless, a peculiar shape of the deviations can be observed for the third flight. A possible explanations for this is connected to the way itself flight guidance is enabled. As said in the introduction, vertical aircraft guidance is based on flight levels, which means that aircraft at cruise altitudes fly along isobar surfaces. These can be more or less detached from surfaces of constant geodetic altitude. 


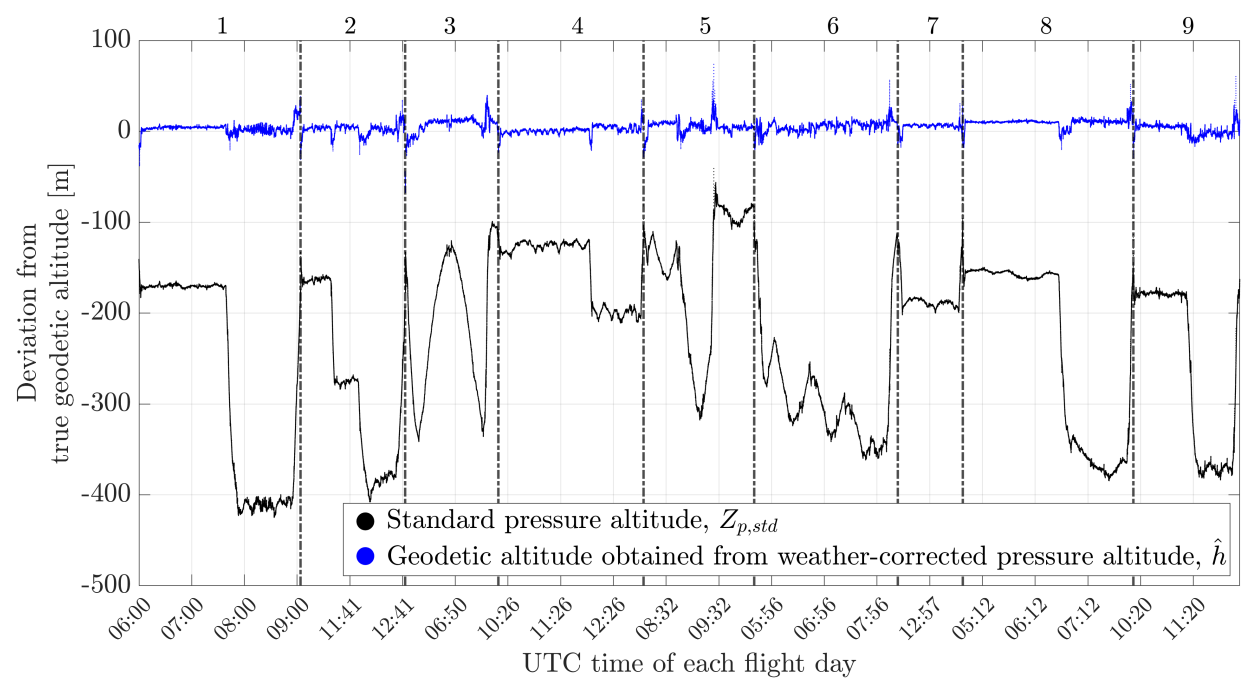

Figure 9: Deviations from the true geodetic altitude of standard pressure altitude and of geodetic altitude obtained from weather-corrected pressure altitude

A certain relation of the altitude deviation with respect to the true geodetic altitude profiles can also be seen in the case of the computed geodetic altitude. In this case, the error increases when there are steep altitude variations, which suggest some dependency of flight dynamics.

\section{Mitigation of dynamics-induced error components}

A linear relationship was found between the altitude errors and the aircraft's pitch angle, as shown in Figure 10 . This corresponds with previous observation that error increases for altitude transitions, since the aircraft changes its attitude during those phases. The inflow direction of air into the pressure ports may be responsible for this correlation.

We derived a linear model relating the errors and the pitch angle by applying a robust linear regression using a bisquare fitting weighting function. The model can be described by $\Delta h(\theta)=m \theta+n$, and the model parameters were found to be $m=-2.44 \mathrm{~m} \mathrm{deg}^{-1}$ and $n=14.47 \mathrm{~m}$. The resulting model is depicted in Figure (10b) for different pitch angles.

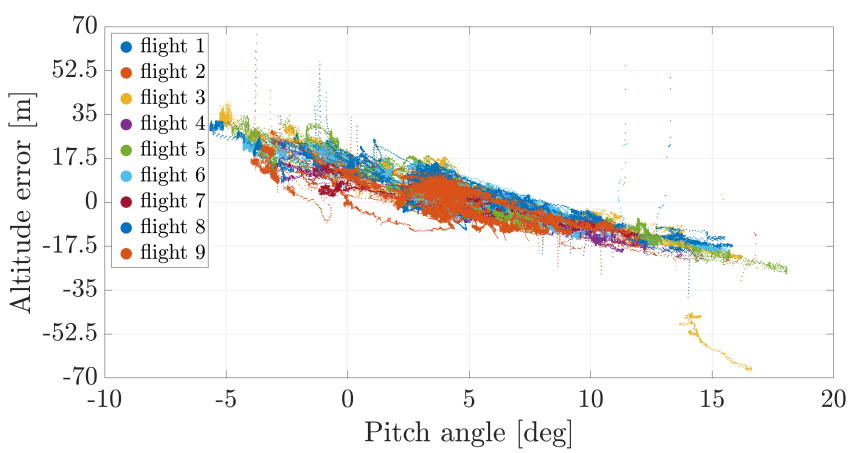

(a) Altitude errors versus the pitch angle of each of the nine flights

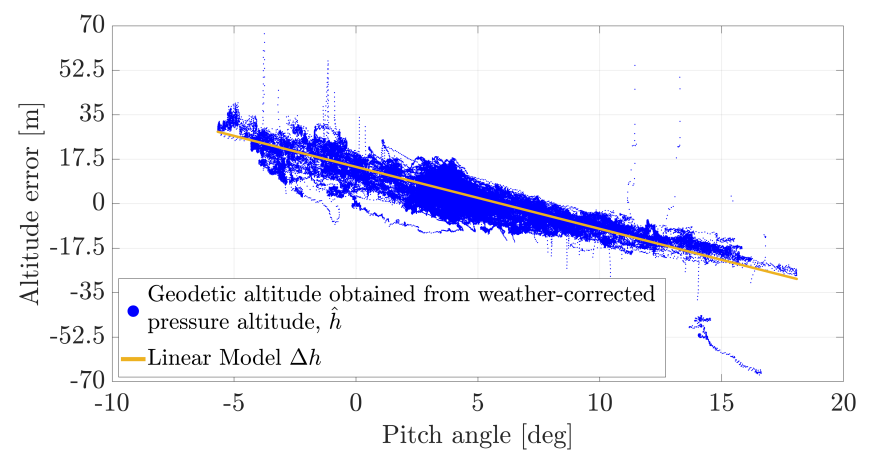

(b) Linear model relating the altitude errors and the pitch angle

Figure 10: Altitude errors and pitch angle 


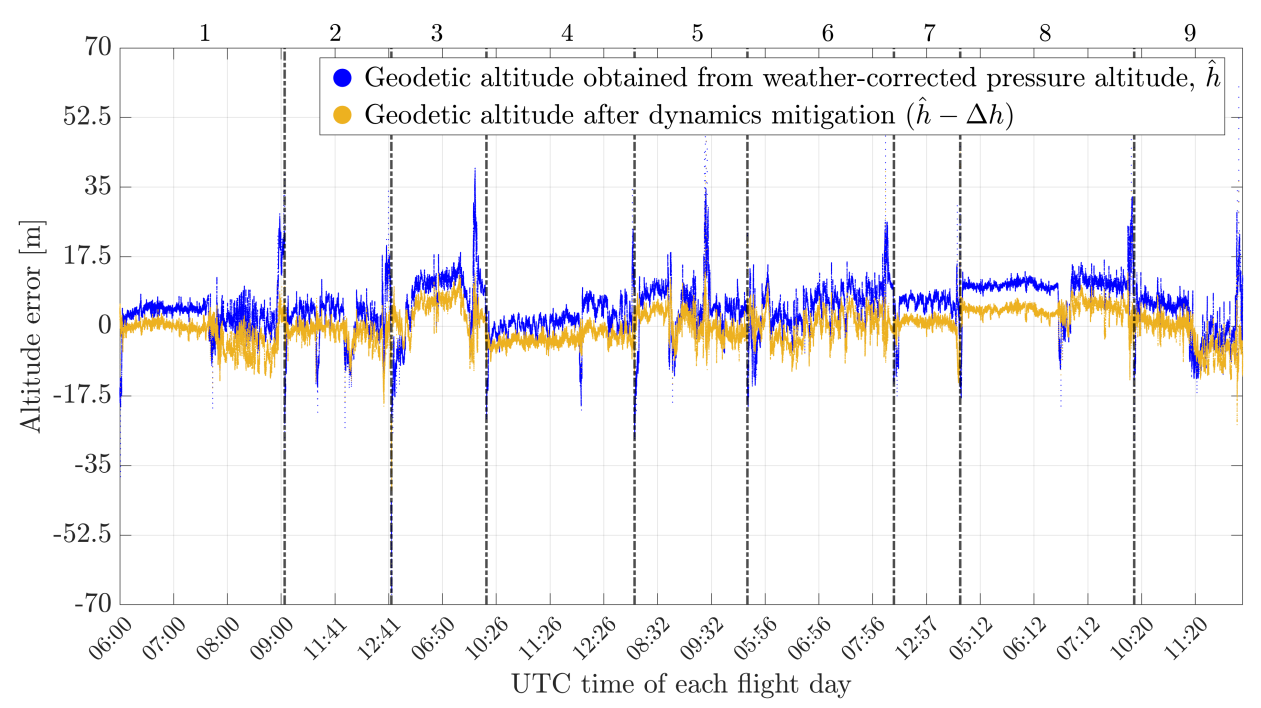

Figure 11: Altitude errors, before and after the mitigation with the linear model based on pitch angle

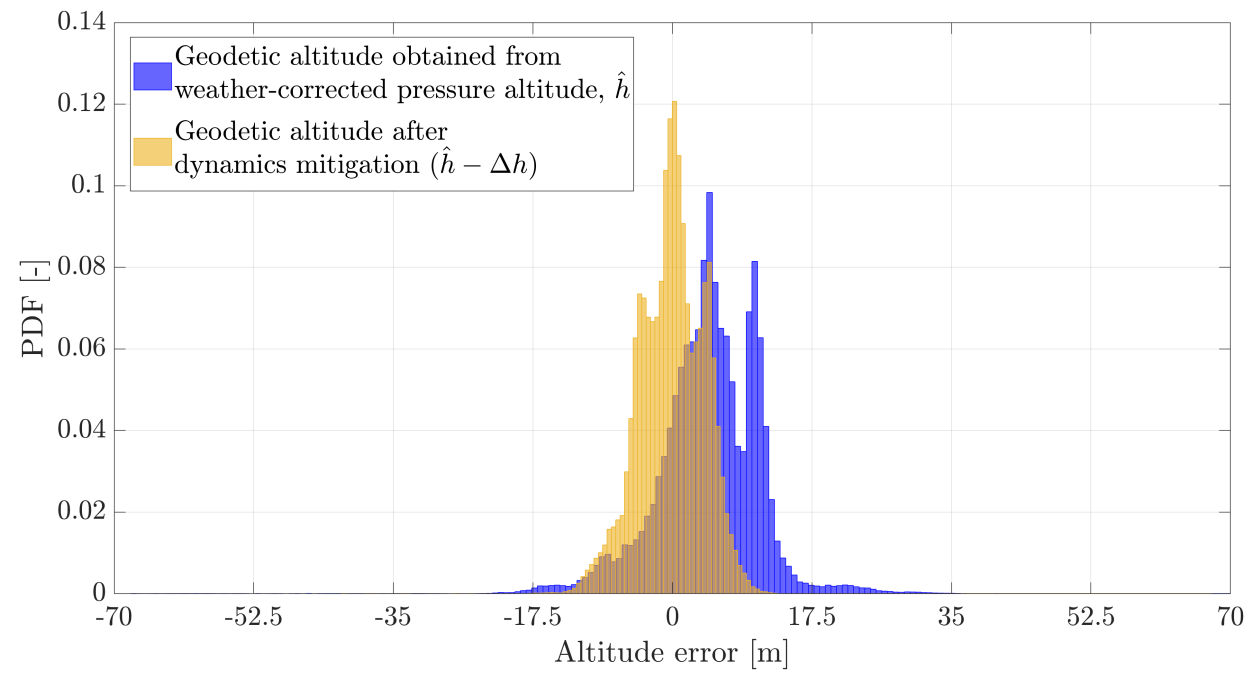

Figure 12: Distributions of the altitude errors, before and after the mitigation

Figure 11 shows the altitude errors before and after applying the correction $\Delta h$ based on pitch angle. From this figure, it can be easily observed that this mitigation is capable of reducing large errors specially during transition phases. Figure 12 shows the probability density function of the error distribution before and after having applied the correction. The values of the mean and of the standard deviation improve from approximately $4.6 \mathrm{~m}$ and $6.4 \mathrm{~m}$, to approximately $-0.1 \mathrm{~m}$ and $4.1 \mathrm{~m}$. respectively.

\section{ROBUST GEODETIC BARO-ALTITUDE ERROR MODELING}

This section addresses the robust statistical modeling of the residual geodetic altitude errors. The analysis are carried out on the geodetic altitude obtained after correction with the linear model of Section V.3.

\section{Gaussian Model for Snapshot Estimators}

Robust positioning algorithms using the computed geodetic altitudes in this work need error models that safely overbounds the underlying residual error. Gaussian error models can be easily considered in linear estimators (e.g. least-squares) because they preserve their distribution through convolution [25]. 


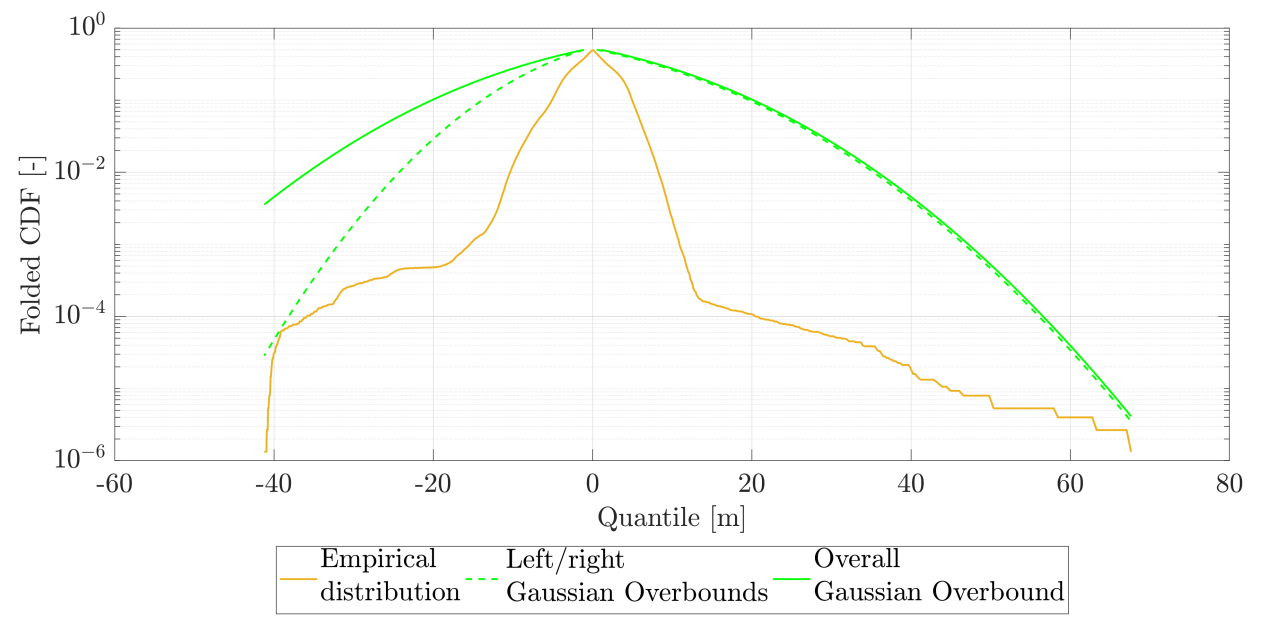

Figure 13: Folded CDFs of Empirical distribution and Gaussian overbounds.

Table 2: Parameters of the paired Gaussian overbound models.

\begin{tabular}{|c||c||c|}
\hline Parameter & Left-hand side & Right-hand side \\
\hline Mean [m] & -1.0717 & 0.527 \\
\hline Standard Deviation [m] & 9.9738 & 14.9362 \\
\hline
\end{tabular}

A Gaussian distribution can therefore be used to bound the residual error distribution. This can be done for instance in the Cumulative Density Function (CDF) sense by using the approach in [25]. In this approach,the left-hand and right-hand side of the Cumulative Density Function (CDF) is independently considered. First, an intermediate symmetrical and unimodal distribution that overbounds the actual distribution in the sense of paired overbounding is computed. Secondly, a Gaussian distribution that overbounds the intermediate one is obtained [25]. Figure 13 depicts the folded CDF of the sample data, the left-hand and right-hand overbounding distributions and the overall paired Gaussian bound selected. Table 2 contains the parameters of the two side bounding Gaussian distributions. The final overbounding model is represented by a bias $|b|=1.072$ $\mathrm{m}$ and a standard deviation of $\sigma=14.94 \mathrm{~m}$.

\section{Dynamic Error Model}

In order to incorporate the computed geodetic altitude into a multi-sensor navigation architecture based on sequential estimators, a dynamic model of the error that captures the time correlation of the residual error is needed. In this work, the model is derived from the flight portions where altitude is approximately constant according to Figure 7 and we expect therefore the error processes to be stationary. It is observed that during each of the stationary segments a constant error deviation is present. This constant bias can be estimated by averaging the residual errors over the stationary period. The maximum constant error over the considered stationary data portions is then considered as an additional bias term in the model [26]. After removing the mean residual constant error from each of the segments as shown in Figure 14, we analyse the frequency response of the error. 


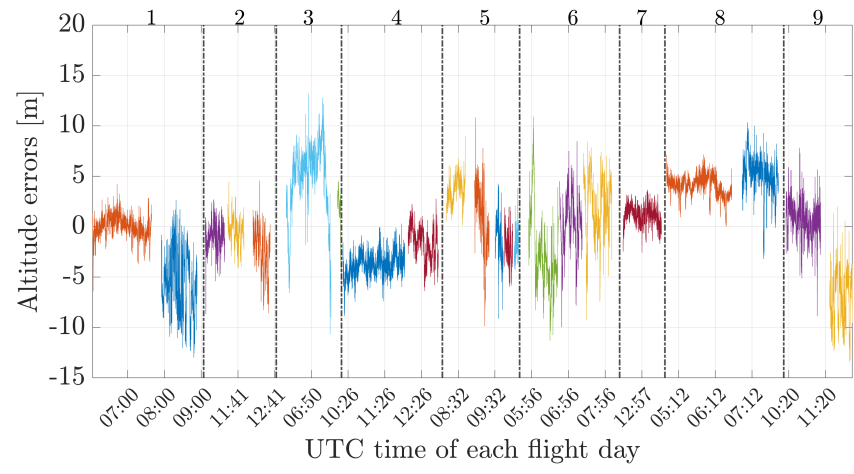

(a) Residual altitude errors

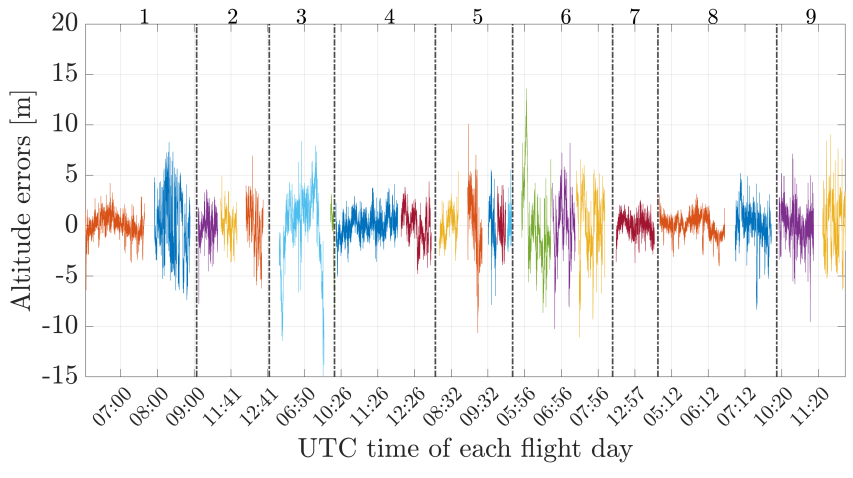

(b) Residual altitude errors along each altitude plateau, about the corresponding mean errors

Figure 14: Evaluation of the residual altitude errors along the constant altitude segments

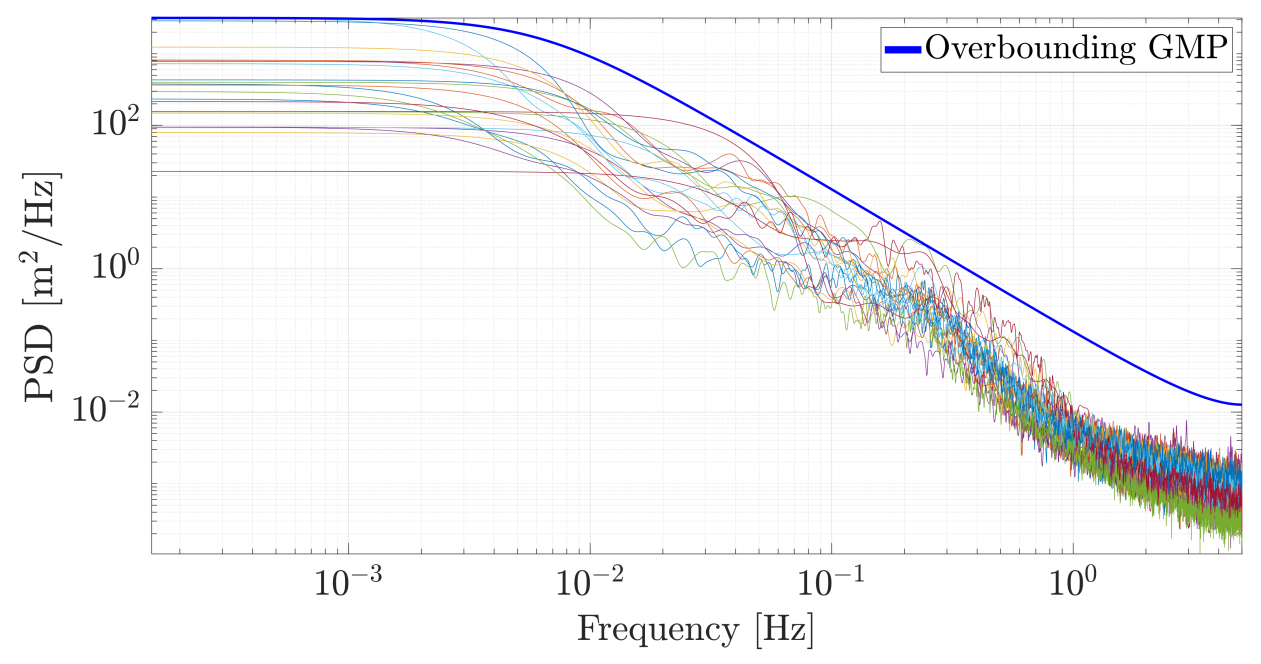

Figure 15: PSD of the residual altitude errors for each altitude plateau and of the overbounding GMP

The Power Spectral Density (PSD) of the residual error is depicted in Figure 15 for the different stationary segments. A dynamic error model that bounds the empirical error in the PSD domain is here proposed since it guarantees bounding estimation conditions when used in sequential estimators [27]. A widely known suitable model is the first-order Gauss-Markov process (GMP). One advantage of such a model is that it could be easily integrated into a Kalman filter (KF) via state augmentation [28]. The power spectral density (PSD) $S(\omega)$ of a discrete-time first-order GMP is [29]:

$$
S(\omega)=\frac{\sigma^{2} \Delta t\left(1-\alpha^{2}\right)}{1+\alpha^{2}-2 \alpha \cos \omega \Delta t},
$$

where $\alpha=e^{\frac{-\Delta t}{\tau}}, \tau$ is the time correlation constant, $\sigma^{2}$ is the stationary variance, $\Delta t$ is the measurement time interval and $\omega$ is the angular frequency. A first-order GMP that bounds all the frequency components of the geodetic altitude errors is found for the parameters $\hat{\tau}=25 \mathrm{~s}$ and $\hat{\sigma}=7.96 \mathrm{~m}$ and is depicted in Figure 15. The final bound consists of a constant bias $b=7 \mathrm{~m}$ together with a GMP that can be described in time domain by:

$$
a_{k}=\hat{\alpha} a_{k-1}+\hat{\sigma} \sqrt{\left(1-\hat{\alpha}^{2}\right)} w_{k},
$$

where $\alpha=e^{-\frac{\Delta t}{\hat{\tau}}}$ and $w_{k} \sim N(0,1)$. 


\section{CONCLUSIONS}

This work assesses the accuracy in the approximation of true geodetic altitude by correcting pressure altitude with external weather data. The consideration of the geoid undulations and the difference between the geopotential and geometric altitude scales enabled a conversion from the corrected pressure altitude to geodetic altitude. This work shows that the geodetic altitude obtained in this way does not show an altitude dependent deviation from the true altitude as compare to the standard pressure altitude. Residual errors were found to contain a linear dependency with the attitude of the aircraft. A linear model is obtained to mitigate this impact based on the aircraft pitch angle. This model allowed to further reduce the absolute value of the mean as well as the standard deviation of the error distribution to $0.1 \mathrm{~m}$ and $4.1 \mathrm{~m}$, respectively.

Secondly, we derived a Gaussian distribution overbounding the residual geodetic altitude errors. This may be suitable for an integration of this altitude information into multi-sensor navigation architectures based on snapshot algorithms. Additionally, we formulated a first-order GMP to dynamically model the residual geodetic altitude errors along the stationary altitude plateaus. This model may be employed when incorporating these geodetic altitude measurements into integrated navigation systems based on sequential estimators.

The investigated flights covered a broad spectrum of altitudes, however measurements from certain altitudes were more numerous. Future work will consider the analysis of larger set of data. In this work we have employed weather data obtained from the Copernicus ERA5 climate reanalysis on pressure levels. In the future, we intend to analyze the impact that different kinds of weather data may have on the weather-corrected pressure altitude computation. Weather forecasts data will be also considered to understand the applicability of this methodology for real time navigation system.

\section{ACKNOWLEDGEMENTS}

The flight data used for the analysis of this work were recorded during the flight campaign of the DLR project ALPS. We would like to thank all the DLR colleagues involved in the coordination and execution of flight trials. We would also like to thank the aeronautics program directorate of DLR for funding this research.

\section{REFERENCES}

[1] Altitude, flight level and height. SKYbrary. Accessed: 2021-06-17. [Online]. Available: https://www.skybrary.aero/ index.php/Altitude,_Flight_Level_and_Height

[2] RTCA, "DO-384 Minimum operational performance standards (MOPS) for GNSS aided inertial systems," Radio Technical Commission for Aeronautics, Tech. Rep. DO384, 2020.

[3] ICAO Doc 9613, Performance Based Navigation Manual, Consoldated 4th Edition, 2013.

[4] G. Berz, V. Vitan, I. Skyrda, and B. Ober, "Can Current DME Support PBN Operations with Integrity?" in Proceedings of the 26th International Technical Meeting of the ION Satellite Division, ION GNSS+, 2013.

[5] O. Osechas, S. Narayanan, O. G. Crespillo, G. Zampieri, G. Battista, R. Kumar, N. Schneckenburger, E. Lay, B. Belabbas, and M. Meurer, "Feasibility demonstration of terrestrial rnp with ldacs," in ION GNSS 2018, November 2019. [Online]. Available: https://elib.dlr.de/131026/

[6] O. Garcia Crespillo, E. Nossek, A. Winterstein, B. Belabbas, and M. Meurer, "Use of High Altitude Platform Systems to Augment Ground Based APNT Systems," in Proceedings of the 34th Digital Avionics Systems Conference (DASC), 9 2015.

[7] O. Garcia Crespillo, A. Grosch, E. Nossek, O. Osechas, B. Belabbas, and M. Meurer, "Integrated inertial Navigation System with Multiple APNT Ranges: Expected Performance and Considerations," in Integrated Communication, Navigation, \& Surveillance Conference (ICNS), Herndon, VA, USA, apr 2016.

[8] E. Nossek, M. Suess, B. Belabbas, and M. Meurer, "Analysis of Position and Timing Solutions for an APNT-System - A look on Convergence, Accuracy and Integrity," in Proceedings of the 2014 ION GNSS+, 2014.

[9] G. Zampieri, S. Narayanan, O. G. Crespillo, and O. Osechas, "A regularized least squares estimator for pseudorange-based terrestrial positioning under degraded geometries," in Proceedings of the 33rd International Technical Meeting of the Satellite Division of The Institute of Navigation (ION GNSS+ 2020), September 2020. [Online]. Available: https://elib.dlr.de/139462/

[10] C. Torens, A. Volkert, D. Becker, D. Gerbeth, L. Schalk, O. G. Crespillo, C. Zhu, T. Stelkens-Kobsch, T. Gehrke, I. C. Metz, and J. Dauer, HorizonUAM: Safety and Security Considerations for Urban Air Mobility. [Online]. Available: https://arc.aiaa.org/doi/abs/10.2514/6.2021-3199 
[11] P. D. Groves, Principles of GNSS, Inertial, and Multisensor Integrated Navigation Systems. Artec House, 2013.

[12] L. Daniel Macias-Valadez; Rock Santerre; Sophie Larochelle; René, "Improving vertical GPS precision with a GPS-overfiber architecture and real-time relative delay calibration," GPS Solutions, vol. 16, no. 4, pp. 449-462, 2012.

[13] T.-C. Li and V. Chueh, "Errors in the pressure and blanchard altitudes for a cross country flight," in Position Location and Navigation Symposium (PLANS), 2010 IEEE/ION, 2010.

[14] S. Gaglione, A. Angrisano, G. Castaldo, C. Gioia, A. Innac, L. Perrotta, G. D. Core, and S. Troisi, "GPS/Barometer augmented navigation system: Integration and integrity monitoring," in 2015 IEEE Metrology for Aerospace (MetroAeroSpace), 2015.

[15] C. Hajiyev, U. Hacizade, and D. Cilden-Guler, "Integration of barometric and GPS altimeters via adaptive data fusion algorithm," International Journal of Adaptive Control and Signal Processing, vol. 35, no. 1, pp. 2-14, 2021.

[16] D. S. Jan, Gebre-Egziabher, T. Walter, and P. Enge, "Improving GPS-Based Landing System Performance using an Empirical Barometric Altimeter Confidence Bound," IEEE Transactions on Aerospace and Electronic Systems, vol. 44, no. 1, 2008.

[17] J. Lee, E. Hyeon, M. Kim, and J. Lee, "VERTICAL POSITION ERROR BOUNDING FOR INTEGRATED GPS / BAROMETER SENSORS TO SUPPORT UNMANNED AERIAL VEHICLE ( UAV )," in 30th Congress of the International Council of the Aeronautical Sciences (ICAS), 2016.

[18] R. L. Blanchard, "A New Algorithm for Computing Inertial Altitude and Vertical Velocity," IEEE Transactions on Aerospace and Electronic Systems, vol. AES-7, no. 6, pp. 1143-1146, 1971.

[19] R. L. Blanchard", "An Improvement to an Algorithm for Computing Aircraft Reference Altitude," IEEE Transactions on Aerospace and Electronic Systems, vol. AES-8, no. 5, pp. 685-687, 1972.

[20] What is the geoid? National Geodetic Survey. Accessed: 2021-06-15. [Online]. Available: https: //geodesy.noaa.gov/GEOID/geoid_def.html

[21] B. Scherllin-Pirscher, A. K. Steiner, G. Kirchengast, M. Schwärz, and S. S. Leroy, "The power of vertical geolocation of atmospheric profiles from GNSS radio occultation," Journal of Geophysical research: atmospheres, vol. 122, no. 1, pp. 1595-1616, 2017.

[22] A. Giez, C. Mallaun, M. Zöger, A. Dörnbrack, and U. Schumann, "Static Pressure from Aircraft Trailing-Cone Measurements and Numerical Weather-Prediction Analysis," Journal of Aircraft, vol. 54, no. 5, 2017.

[23] International Civil Aviation Organization, Manual of ICAO Standard Atmosphere, extended to 80 kilometres (262500 feet), Document 7488/3, 1993.

[24] H. Hersbach, B. Bell, P. Berrisford, A. H. G. Biavati, J. M. Sabater, J. P. C. Nicolas, R. Radu, I. Rozum, D. Schepers, A. Simmons, C. Soci, D. Dee, and J.-N. Thépaut, "ERA5 hourly data on pressure levels from 1979 to present," Copernicus Climate Change Service (C3S) Climate Data Store (CDS), accessed: 2021-06-15.

[25] J. Blanch, T. Walter, and P. Enge, "Gaussian bounds of sample distributions for integrity analysis," IEEE Transactions on Aerospace and Electronic Systems, vol. 55, no. 4, pp. 1806-1815, 2019.

[26] E. Gallon, M. Joerger, and B. Pervan, "Frequency-domain modeling of orbit and clock errors for sequential positioning," 102020.

[27] S. Langel, O. García Crespillo, and M. Joerger, "A New Approach for Modeling Correlated Gaussian Errors using Frequency Domain Overbounding," in Position, Navigation and Timing Symposium (PLANS), 52020.

[28] S. Langel, O. García Crespillo, and M. Joerger, "Overbounding the effect of uncertain gauss-markov noise in kalman filtering," NAVIGATION, vol. 68, no. 2, pp. 259-276, 2021. [Online]. Available: https://onlinelibrary.wiley.com/doi/abs/10.1002/navi.419

[29] O. G. Crespillo, M. Joerger, and S. Langel, "Tight bounds for uncertain time-correlated errors with gauss-markov structure," arXiv, 2020. 